\title{
Investigating the functional-compulsive continuum in animals: Contextual lever-pressing in the deer mouse model of obsessive-compulsive disorder (OCD)
}

\author{
De Wet Wolmarans ${ }^{1}$, Geoffrey De Brouwer ${ }^{1}$, Brian H Harvey ${ }^{1,2}$ \\ ${ }^{I}$ Division of Pharmacology, North-West University, Potchefstroom, South Africa, ${ }^{2}$ MRC Unit on Risk and Resilience in \\ Mental Disorders, South Africa
}

Introduction

Conceptualizing animal models of OCD is problematic; compulsions are believed to be driven by goal-directed triggers borne from obsessive thinking, e.g. contamination fears and washing compulsions, while deficits in reward feedback are proposed to perpetuate failure of task completion leading to said aberrant behavior. Here we attempt to elucidate the contextual triggers of compulsive-like behavior, by observing responses in outcome-feedback processing in large nesting deer mice, a validated model of OCD. Applying the natural need to nest as a trigger for compulsivity, we aimed to demonstrate that goal-directed triggers underlie the funcional-compulsive continuum.

Methods

20 deer mice (Peromyscus maniculatus bairdii) of both sexes were classified into into normal $(\mathrm{NNB} ; \mathrm{n}=10)$ and large nesting (LNB; $n=10)$ groups. Subsequently, they were operant-trained (coincidentally) over one week to press a homecage installed lever to obtain $20 \mathrm{~mm}$ of nesting material per press (response to a lever press lasts $1 \mathrm{~s}$ ). Over 24 hrs animals were allowed 22 hours with lever-presses, leaving 2 hours to remove built nests and clean cages. Thereafter, this was repeated for another week during which time the daily number of lever-presses was recorded to establish a baseline nesting score for every individual. During the third week, animals again pressed levers in the absence of nesting-material to assess outcome-feedback processing.

Results

LNB deer mice present with significantly increased ritualized behavior compared to NNB animals, as measured by the number of lever-presses executed in the absence of the expected outcome (i.e. nesting material) $(p<0.05)$. Further, NNB mice are significantly quicker than LNB deer mice in appreciating the devaluation of reward by ceasing to press levers in the absence of a reward $(\mathrm{p}<0.05)$.

Conclusion

We present evidence in a rodent model of OCD that seemingly purposeless repetitions in animals can initially be triggered by context-driven motivation, even in the absence of a expected reward. Thus, the findings reported here elaborate our understanding of repetitive behavior in animal models of OCD, by demonstrating a contextual foundation for the transition of goal-directed to habitual behaviour in an animal model of OCD. 